T. Shiga, A. Shimizu and T. Soshi

Nagoya Math. J.

Vol. 162 (2001), 169-185

\title{
PASSAGE-TIME MOMENTS FOR POSITIVELY RECURRENT MARKOV CHAINS
}

\author{
TOKUZO SHIGA ${ }^{1}$, AKINOBU SHIMIZU ${ }^{2}$ AND \\ TAKAHIRO SOSHI
}

\begin{abstract}
Fractional moments of the passage-times are considered for positively recurrent Markov chains with countable state spaces. A criterion of the finiteness of the fractional moments is obtained in terms of the convergence rate of the transition probability to the stationary distribution. As an application it is proved that the passage time of a direct product process of Markov chains has the same order of the fractional moments as that of the single Markov chain.
\end{abstract}

\section{$\S 1$. Problems and results}

In this paper we are concerned with the fractional moments of the passage-time for continuous time Markov chains. The problem of finding criteria for the finiteness and infiniteness of the passage-time moments has been studied by many authors in various situations both for Markov or nonMarkov processes. (eg. [AI], [L], [TT], [CK], [MW] etc.) However most of these results give only sufficient conditions for the finiteness and infiniteness of the passage-time moments.

It has been well-recognized that the finiteness of the fractional moments is closely related to convergence rate of the transition probability to the stationary distribution for positively recurrent Markov processes and to its decay rate for null recurrent Markov processes, but it seems not straightforward to describe their relations.

Let $S$ be a countable set, and let $\left(X_{t}, P_{x}\right)_{t \geq 0, x \in S}$ be a continuous time Markov chain with state space $S$. The transition probabilities of the Markov chain are denoted by $p_{t}(x, y), x, y \in S$. Throughout this paper we assume that $\left(X_{t}, P_{x}\right)_{t \geq 0, x \in S}$ is irreducible and positively recurrent. Then the in-

Received May 24, 1999.

Revised April 14, 2000.

2000 Mathematics Subject Classification: 60J27.

${ }^{1}$ Supported in part by Grant-in-Aid for Scientific Research 09640246.

${ }^{2}$ Supported in part by Grant-in-Aid for Scientific Research 10640130. 
finitesimal matrix $\left\{q_{x, y}\right\}_{x, y \in S}$ of the transition probability satisfies

$$
q_{x, y} \geq 0 \quad(x \neq y), \quad \sum_{y \in S} q_{x, y}=0 \quad(y \in S),
$$

and

$$
0<q_{x}=-q_{x, x}<\infty \quad(x \in S) .
$$

For $x \in S$ let $T_{x}$ be the passage-time to $x$, and denote by $T$ the first returning time, namely

$$
T_{x}=\inf \left\{t \geq 0 ; X_{t}=x\right\}
$$

and

$$
T=\inf \left\{t>0 ; X_{t}=X_{0} \quad \text { and } \quad X_{s} \neq X_{0} \quad \text { for } \quad \text { some } \quad s<t\right\}
$$

Under the present assumption it is known that $E_{x}(T)<\infty$ holds for every $x \in S$ and there exists a unique stationary distribution $\nu=\left(\nu_{x}\right)$ which is given by

$$
\nu_{x}=\frac{1}{q_{x} E_{x}(T)} \quad(x \in S) .
$$

As seen in Lemma 2.1, to investigate the fractional moments of the passagetimes it suffices to consider the fractional moments of the first returning time to any fixed starting point.

In the present paper we give a complete criterion for the finiteness and infiniteness of the first returning time fractional moments in terms of convergence rate of the transition probability to the stationary distribution as $t \rightarrow \infty$.

One of our motivations comes from a problem arising in population genetics. In $[\mathrm{SS}]$ was obtained a limit theorem involving the mean number of different types in $n$ random sampling in a certain stationary interactive Fleming-Viot processes under the assumption that for the $n$-product process $\left(\boldsymbol{X}_{t}, \boldsymbol{P}_{\mathbf{X}}^{(n)}\right)$ of the Markov chain $\left(X_{t}, P_{x}\right)_{t \geq 0, x \in S}, \boldsymbol{E}_{\mathbf{X}}^{(n)}\left(\boldsymbol{T}^{2}\right)<\infty$ is fulfilled, where $\boldsymbol{T}$ stands for the first returning time of $\left(\boldsymbol{X}_{t}, \boldsymbol{P}_{\mathbf{X}}^{(n)}\right)$, so that the following problem arises naturally:

Let $\alpha$ be a real number with $\alpha \geq 1$ and $n$ be an integer with $n \geq 2$. Then does it hold that $E_{x}\left(T^{\alpha}\right)<\infty$ implies $\boldsymbol{E}_{\mathbf{X}}^{(n)}\left(\boldsymbol{T}^{\alpha}\right)<\infty$ ? 
In the case $\alpha=1$, the problem is trivial, because the $n$-product process is also positively recurrent and its stationary distribution $\nu^{(n)}=\left(\nu^{(n)}(\mathbf{x})\right)$ is given by

$$
\nu^{(n)}(\mathbf{x})=\Pi_{j=1}^{n} \nu_{x_{j}}, \quad \text { for } \quad \mathbf{x}=\left(x_{1}, x_{2}, \cdots, x_{n}\right) \in S^{n} .
$$

However if $\alpha>1$, the problem is highly non-trivial. In this paper we present two theorems which give criteria for the finiteness and infiniteness of the fractional moments, and applying these results we solve the above problem in Theorem 1.3.

Our result are the following.

TheOrem 1.1. Let $\alpha>1$. Then $E_{x}\left(T^{\alpha}\right)<\infty$ if and only if

$$
\sup _{\lambda>0} \int_{0}^{\infty} e^{-\lambda t} t^{\alpha-2}\left(p_{t}(x, x)-\nu_{x}\right) d t<\infty .
$$

For $\beta>-1$, let

$$
r_{\lambda}^{(\beta)}(0)=\int_{0}^{\infty} e^{-\lambda t} t^{\beta}\left(p_{t}(x, x)-\nu_{x}\right) d t .
$$

It should be noted that $r_{\lambda}^{(\beta)}(0) \geq 0$ does not hold in general, (see Remark 2.1). However if (1.2) is fulfilled, then

$$
\lim _{\lambda \searrow 0} r_{\lambda}^{(\alpha-2)}(0)
$$

exists as seen in the proof of Theorem 1.1.

Next we state another version of the fractional moment criterion. Let $\left(\left(X_{t}, Y_{t}\right), P_{x} \otimes P_{y}\right)$ be the direct product process of $\left(X_{t}, P_{x}\right)$, and we denote by $T$ and $T^{\prime}$ the first returning times of $X_{t}$ and $Y_{t}$.

Theorem 1.2. (i) For $\beta>-1$,

$$
E_{x} \otimes E_{x}\left(T T^{\prime}\left(T \wedge T^{\prime}\right)^{\beta+1}\right)<\infty
$$

holds if and only if

$$
\int_{1}^{\infty} t^{\beta}\left(p_{t}(x, x)-\nu_{x}\right)^{2} d t<\infty
$$


(ii) For $\beta=-1$,

$$
E_{x} \otimes E_{x}\left(T T^{\prime} \log _{+}\left(T \wedge T^{\prime}\right)\right)<\infty
$$

holds if and only if

$$
\int_{1}^{+\infty} t^{-1}\left(p_{t}(x, x)-\nu_{x}\right)^{2} d t<\infty
$$

We remark that if $E\left(T^{(\beta+3) / 2}\right)<\infty,(1.4)$ does hold, but the converse is not true in general. If (1.4) is fulfilled, it holds that $E\left(T^{\gamma}\right)<\infty$ for any $0<\gamma<(\beta+3) / 2$, (see Lemma 4.1).

Let $\left(X_{t}^{1}, P_{x_{1}}^{1}\right)$ and $\left(X_{t}^{2}, P_{x_{2}}^{2}\right)$ be two positively recurrent and irreducible Markov chains with state spaces $S^{1}$ and $S^{2}$, and let $T^{1}$ and $T^{2}$ the first returning times of $\left(X_{t}^{1}, P_{x_{1}}^{1}\right)$ and $\left(X_{t}^{2}, P_{x_{2}}^{2}\right)$, respectively. We denote by $\boldsymbol{T}$ the first returning time of the product process $\left.\left(\left(X_{t}^{1}, X_{t}^{2}\right), P_{x_{1}}^{1} \otimes P_{x_{2}}^{2}\right)\right)$.

TheOREM 1.3. Let $\alpha>1$, and $x_{1} \in S^{1}, x_{2} \in S^{2}$. Suppose that $E_{x_{1}}^{1}\left(\left(T^{1}\right)^{\alpha}\right)<\infty$ and $E_{x_{2}}^{2}\left(\left(T^{2}\right)^{\alpha}\right)<\infty$, then it holds $E_{x_{1}}^{1} \otimes E_{x_{2}}^{2}\left(\boldsymbol{T}^{\alpha}\right)<\infty$.

Theorem 1.3 follows from Theorem 1.1 and Theorem 1.2. In fact, denot$\operatorname{ing} p_{t}^{1}\left(x_{1}, y_{1}\right), p_{t}^{2}\left(x_{2}, y_{2}\right), \nu^{1}=\left(\nu_{x_{1}}^{1}\right)$ and $\nu^{2}=\left(\nu_{x_{2}}^{2}\right)$ the transition probabilities and the stationary distributions of $\left(X_{t}^{1}, P_{x_{1}}^{1}\right)$ and $\left(X_{t}^{2}, P_{x_{2}}^{2}\right)$ respectively, by Theorem 1.1 it suffices to show that

$$
\sup _{\lambda>0} \int_{0}^{\infty} e^{-\lambda t} t^{\alpha-2}\left(p_{t}^{1}\left(x_{1}, x_{1}\right) p_{t}^{2}\left(x_{2}, x_{2}\right)-\nu_{x_{1}}^{1} \nu_{x_{2}}^{2}\right) d t<\infty
$$

However from the assumption and Theorem 1.1 it holds

$$
\sup _{\lambda>0} \int_{0}^{\infty} e^{-\lambda t} t^{\alpha-2}\left(p_{t}^{i}\left(x_{i}, x_{i}\right)-\nu_{x_{i}}^{i}\right) d t<\infty \quad(i=1,2)
$$

and by Theorem 1.2 with $\beta=2 \alpha-3$ it holds

$$
\int_{1}^{\infty} t^{\beta}\left(p_{t}^{i}(x, x)-\nu_{x_{i}}^{i}\right)^{2} d t<\infty \quad(i=1,2)
$$


so that (1.10) is valid for $\beta=\alpha-2$ because of $\alpha>1$. Now note that

$$
\begin{aligned}
& p_{t}^{1}\left(x_{1}, x_{1}\right) p_{t}^{2}\left(x_{2}, x_{2}\right)-\nu_{x_{1}}^{1} \nu_{x_{2}}^{2} \\
\leq & \left(p_{t}^{1}\left(x_{1}, x_{1}\right)-\nu_{x_{1}}^{1}\right) \nu_{x_{2}}^{2}+\left(p_{t}^{2}\left(x_{2}, x_{2}\right)-\nu_{x_{2}}^{2}\right) \nu_{x_{1}}^{1} \\
& +\frac{1}{2}\left(p_{t}^{1}\left(x_{1}, x_{1}\right)-\nu_{x_{1}}^{1}\right)^{2}+\frac{1}{2}\left(p_{t}^{2}\left(x_{2}, x_{2}\right)-\nu_{x_{2}}^{2}\right)^{2}
\end{aligned}
$$

Combining this with (1.9) and (1.10) with $\beta=\alpha-2$ we obtain (1.8), which completes the proof of Theorem 1.3.

\section{$\S 2$. Preliminary lemmas}

Lemma 2.1. Suppose that the Markov chain $\left(X_{t}, P_{x}\right)$ is irreducible. Then for $\alpha>0$, the following three are equivalent.

(i) $E_{x}\left(T^{\alpha}\right)<\infty$ for some $x \in S$.

(ii) $\quad E_{x}\left(T^{\alpha}\right)<\infty$ for every $x \in S$.

(iii) $E_{x}\left(T_{y}^{\alpha}\right)<\infty$ for every $x \neq y \in S$.

Proof. Obviously (i) and irreducibility imply $E_{y}\left(T_{x}^{\alpha}\right)<\infty$ for every $y \in S$. Next we claim that

$$
E_{x}\left(T_{y}^{\alpha}\right)<\infty \text { for every } y \in S
$$

Using the following inequality; for any $\varepsilon>0$ there exists a $C(\varepsilon)>0$ such that

$$
(a+b)^{\alpha} \leq(1+\varepsilon) a^{\alpha}+C(\varepsilon) b^{\alpha} \quad(a>0, b>0)
$$

and the strong Markov property, we see that for every $M>0$

$$
\begin{aligned}
E_{x}\left(T_{y}^{\alpha} \wedge M\right) & =E_{x}\left(T_{y}^{\alpha} \wedge M: T_{y}<T\right)+E_{x}\left(T_{y}^{\alpha} \wedge M: T_{y}>T\right) \\
& \leq E_{x}\left(T^{\alpha}\right)+E_{x}\left(\left(T+\theta_{T} \cdot T_{y}\right)^{\alpha} \wedge M: T_{y}>T\right) \\
& \leq(1+C(\varepsilon)) E_{x}\left(T^{\alpha}\right)+(1+\varepsilon) P_{x}\left(T_{y}>T\right) E_{x}\left(T_{y}^{\alpha} \wedge M\right)
\end{aligned}
$$

Noting that $P_{x}\left(T_{y}>T\right)<1$ due to the irreducibility, choose $\varepsilon>0$ so that $(1+\varepsilon) P_{x}\left(T_{y}>T\right)<1$, then $(2.1)$ is immediate from $(2.2)$.

The rest is trivial since for $x, y \in S, E_{x}\left(T_{y}^{\alpha}\right)<\infty$ and $E_{y}\left(T_{x}^{\alpha}\right)<\infty$ imply $E_{x}\left(T^{\alpha}\right)<\infty$ and $E_{y}\left(T^{\alpha}\right)<\infty$. 
Let $x \in S$ be fixed. For $\lambda>0$ and $\alpha>-1$, let us introduce the following functions of $z \in \boldsymbol{R}$ :

$$
\begin{gathered}
r_{\lambda}^{(\alpha)}(z)=\int_{0}^{\infty} e^{-(\lambda+i z) t} t^{\alpha}\left(p_{t}(x, x)-\nu_{x}\right) d t \\
\varphi_{\lambda}^{(\alpha)}(z)=E_{x}\left(\int_{0}^{T} e^{-(\lambda+i z) t} t^{\alpha} d t\right), \\
G_{\lambda}^{(\alpha)}(z)=\int_{0}^{\infty} e^{-(\lambda+i z) u} u^{\alpha} g(u) d u
\end{gathered}
$$

where

$$
g(u)=\nu_{x}\left(\left(1-e^{-q_{x} u}\right) E_{x}(T)-E_{x}(T \wedge u)\right)
$$

In particular we denote $r_{\lambda}(z)=r_{\lambda}^{(0)}(z)$ and $\varphi_{\lambda}(z)=\varphi_{\lambda}^{(0)}(z)$. Notice that $r_{\lambda}^{(\alpha)}, \varphi_{\lambda}^{(\alpha)}$, and $G_{\lambda}^{(\alpha)}$ are complex-valued functions, but $r_{\lambda}^{(\alpha)}(0), \varphi_{\lambda}^{(\alpha)}(0)$ and $\psi_{\lambda}^{(\alpha)}(0)$ are real.

The proofs of Theorems 1.1 and 1.2 are based on the following relations.

\section{LEMMA 2.2.}

(i)

$$
r_{\lambda}(z) \varphi_{\lambda}(z)=G_{\lambda}(z)
$$

(ii) For integer $n \geq 1$

$$
\sum_{k=0}^{n}\left(\begin{array}{l}
n \\
k
\end{array}\right) r_{\lambda}^{(k)}(z) \varphi_{\lambda}^{(n-k)}(z)=G_{\lambda}^{(n)}(z)
$$

(iii) For integer $n \geq 0$ and $0<\gamma<1$,

$$
\sum_{k=0}^{n}\left(\begin{array}{l}
n \\
k
\end{array}\right)\left(r_{\lambda}^{(k+\gamma)}(z) \varphi_{\lambda}^{(n-k)}(z)+B_{\lambda}^{n, k, \gamma}(z)\right)=G_{\lambda}^{(n+\gamma)}(z)
$$

where for $0 \leq k \leq n$ and $0<\gamma<1$

$$
B_{\lambda}^{n, k, \gamma}(z)=\frac{\gamma}{\Gamma(1-\gamma)} \int_{0}^{\infty} \frac{r_{\lambda+y}^{(k)}(z)\left(\varphi_{\lambda}^{(n-k)}(z)-\varphi_{\lambda+y}^{(n-k)}(z)\right)}{y^{\gamma+1}} d y
$$


Proof. Let $T^{(n)}$ be the $n$-th returning time, and $\zeta$ be the first jump time. Since the distribution of $\zeta$ under $P_{x}$ be the exponential distribution with parameter $q_{x}$, by using the strong Markov property we have

$$
\begin{aligned}
E_{x}\left(\int_{T^{(n)}}^{T^{(n+1)}} e^{-(\lambda+i z) t} I(X(t)=x) d t\right) & =E_{x}\left(e^{-(\lambda+i z) T^{(n)}}\right) \frac{1}{\lambda+q_{x}+i z} \\
& =\frac{\left(E_{x}\left(e^{-(\lambda+i z) T}\right)\right)^{n}}{\lambda+q_{x}+i z}
\end{aligned}
$$

which implies

$$
\int_{0}^{\infty} e^{-(\lambda+i z) t} p_{t}(x, x) d t=\frac{1}{\varphi_{\lambda}(z)(\lambda+i z)\left(\lambda+q_{x}+i z\right)} .
$$

Using (1.1), we have

$$
r_{\lambda}(z) \varphi_{\lambda}(z)=\frac{\nu_{x}}{\lambda+i z}\left(\frac{q_{x} E_{x}(T)}{\lambda+q_{x}+i z}-\varphi_{\lambda}(z)\right),
$$

and the r.h.s. is easily identified with $G_{\lambda}(z)$, which proves (2.7). To see (2.8) take $n$ times differentiations in $\lambda>0$ for both hand sides of (2.7). (2.9) is easily checked using (2.8) and the equality

$$
\frac{\gamma}{\Gamma(1-\gamma)} \int_{0}^{\infty} \frac{1-e^{-y u}}{y^{\gamma+1}} d y=u^{\gamma}
$$

Lemma 2.3. (i) There exist constants $c_{1}>0$ and $c_{2}>0$ such that

$$
\frac{c_{1}}{1+\lambda+|z|} \leq\left|\varphi_{\lambda}(z)\right| \leq \frac{c_{2}}{1+\lambda+|z|} \quad(\lambda>0, z \in \boldsymbol{R}) .
$$

In particular,

$$
\frac{c_{1}}{1+\lambda} \leq \varphi_{\lambda}(0) \leq \frac{c_{2}}{1+\lambda} \quad(\lambda>0)
$$

(ii) If $E_{x}\left(T^{\alpha+1}\right)<+\infty$ for $\alpha>0, \frac{\varphi_{\lambda}^{(\alpha)}(z)}{\varphi_{\lambda}(z)}$ is bounded in $\lambda>0$ and $z \in \boldsymbol{R}$. 
Proof. Note that by the strong Markov property

$$
\varphi_{\lambda}(z)=\frac{1}{\lambda+i z} E_{x}\left(1-E_{X_{\zeta}}\left(e^{-(\lambda+i z) T_{x}}\right) \frac{q_{x}}{\lambda+q_{x}+i z}\right)
$$

which implies that

$$
\lim _{\lambda+|z| \rightarrow \infty}|1+\lambda+i z|\left|\varphi_{\lambda}(z)\right|=1
$$

and

$$
\inf _{\lambda>0, z \in R}|1+\lambda+i z|\left|\varphi_{\lambda}(z)\right|>0
$$

yielding (2.12).

Next, note that

$$
\varphi_{\lambda}^{(\alpha)}(z)=\frac{-1}{\lambda+i z} E_{x}\left(e^{-(\lambda+i z) T} T^{\alpha}\right)+\frac{1}{\lambda+i z} E_{x}\left(\int_{0}^{T} e^{-(\lambda+i z) u} \alpha u^{\alpha-1} d u\right),
$$

from which it follows

$$
\left|\varphi_{\lambda}^{(\alpha)}(z)\right| \leq \frac{2}{|\lambda+i z|} E_{x}\left(T^{\alpha}\right)
$$

Noting that

$$
\lim _{\lambda+|z| \rightarrow 0} \frac{\varphi_{\lambda}^{(\alpha)}(z)}{\varphi_{\lambda}(z)}=\frac{E_{x}\left(T^{\alpha+1}\right)}{E_{x}(T)},
$$

which yields (ii) by (2.12).

LEMMA 2.4. Let $-1<\beta<\alpha$.

(i) If $\sup _{\lambda>0} r_{\lambda}^{(\alpha)}(0)<\infty$, then $\sup _{\lambda>0} r_{\lambda}^{(\beta)}(0)<\infty$.

(ii) If $-1<\alpha \leq 0$, then $r_{\lambda}^{(\alpha)}(0)>0$ for $\lambda>0$.

Proof. Note that

$$
\begin{aligned}
\int_{0}^{1} y^{\alpha-\beta-1} r_{\lambda+y}^{(\alpha)}(0) d y & =\Gamma(\alpha-\beta) r_{\lambda}^{(\beta)}(0) \\
& -\int_{0}^{\infty} e^{-\lambda t} t^{\beta}\left(\int_{t}^{\infty} u^{\alpha-\beta-1} e^{-u} d u\right)\left(p_{t}(x, x)-\nu_{x}\right) d t .
\end{aligned}
$$

Since the 1.h.s. is bonded in $\lambda>0$ by the assumption and so is the last term of the r.h.s., (i) holds. 
For (ii) use (1.1) and the strong Markov property, then

$$
r_{\lambda}(0)=\frac{q_{x} E_{x}\left(E_{X_{\zeta}}\left(e^{-\lambda T_{x}}-1+\lambda T_{x}\right)\right)}{\lambda q_{x}\left(\lambda+q_{x}\right) E_{x}\left(1-e^{-\lambda T}\right) E_{x}(T)} .
$$

Since $e^{-x}-1+x>0 \quad(x>0)$, we have $r_{\lambda}(0)>0$. Moreover,

$$
r_{\lambda}^{(\alpha)}(0)=\Gamma(-\alpha)^{-1} \int_{0}^{\infty} y^{-\alpha-1} r_{\lambda+y}(0) d y
$$

$\operatorname{implies} r_{\lambda}^{(\alpha)}(0)>0$.

Remark 2.1. $\quad r_{\lambda}^{(n)}(0) \geq 0$ holds for every integer $n \geq 0$ and every $\lambda>0$ if and only if $p_{t}(x, x) \geq \nu_{x}$ for every $t \geq 0$, that is true if $p_{t}(x, y)$ have a reversible measure, but it is not true in general.

\section{$\S 3$. Proof of Theorem 1.1}

We prepare the following lemma.

LEMMA 3.1. Let $\alpha>-1 . E_{x}\left(T^{\alpha+2}\right)<\infty$ if and only if

$$
\sup _{\lambda>0} G_{\lambda}^{(\alpha)}(0)<\infty
$$

Proof. As easily seen,

$$
\sup _{\lambda>0} \int_{0}^{\infty} e^{-\lambda u} E_{x}\left((T-u)_{+}-T e^{-q_{x} u}\right) u^{\alpha} d u<\infty
$$

is equivalent to

$$
\int_{0}^{\infty} E_{x}\left((T-u)_{+}\right) u^{\alpha} d u<\infty
$$

which turns to $E_{x}\left(T^{\alpha+2}\right)<\infty$.

We divide the proof of Theorem 1.1 into 3 cases.

Case 1: $\alpha=n+2\left(n \in \boldsymbol{Z}_{+}\right)$.

By Lemma 2.2 (i)

$$
r_{\lambda}(0) \varphi_{\lambda}(0)=G_{\lambda}(0)
$$

which yields that $\sup _{0<\lambda<1} r_{\lambda}(0)<\infty$ is equivalent to $\sup _{0<\lambda<1} G_{\lambda}(0)<\infty$, since $0<\inf _{0<\lambda<1} \varphi_{\lambda}(0)<\infty$. Moreover by Lemma 3.1 this condition is equivalent to $E_{x}\left(T^{2}\right)<\infty$. Then it is obvious that

$$
\lim _{\lambda \searrow 0} r_{\lambda}(0)=\frac{\lim _{\lambda \searrow 0} G_{\lambda}(0)}{E_{x}(T)} \quad \text { exists. }
$$


Next for $0 \leq k<n$, suppose that $\sup _{\lambda>0} r_{\lambda}^{(k)}(0)<\infty$ is equivalent to $E_{x}\left(T^{k+2}\right)<\infty$ for $0 \leq k<n$, and that

$$
\lim _{\lambda \searrow 0} r_{\lambda}^{(k)}(0) \quad \text { exists. } \quad(0 \leq k<n)
$$

By Lemma 2.2 (ii)

$$
r_{\lambda}^{(n)}(0) \varphi_{\lambda}(0)+\sum_{k=0}^{n-1}\left(\begin{array}{l}
n \\
k
\end{array}\right) r_{\lambda}^{(k)}(0) \varphi_{\lambda}^{(n-k)}(0)=G_{\lambda}^{(n)}(0)
$$

If $\sup _{\lambda>0} r_{\lambda}^{(n)}(0)<\infty$, by Lemma $2.4 \sup _{\lambda>0} r_{\lambda}^{(k)}(0)<\infty$ for $0 \leq k \leq n$, so that by the inductive assumption we know $E_{x}\left(T^{n+1}\right)<\infty$. Hence the l.h.s. of (3.1) is bounded in $\lambda>0$, so that $\sup _{\lambda>0} G_{\lambda}^{(n)}(0)<\infty$, which is equivalent to $E_{x}\left(T^{n+2}\right)<\infty$. The rest of the statement is also proved by the same induction argument, so we skip it.

Case 2: $\alpha=n+2+\gamma\left(n \in Z_{+}, 0<\gamma<1\right)$.

By Lemma 2.2

$$
\begin{aligned}
& r_{\lambda}^{(n+\gamma)}(0) \varphi_{\lambda}(0)+\sum_{k=0}^{n-1}\left(\begin{array}{l}
n \\
k
\end{array}\right) r_{\lambda}^{(k+\gamma)}(0) \varphi_{\lambda}^{(n-k)}+\sum_{k=0}^{n}\left(\begin{array}{l}
n \\
k
\end{array}\right) B_{\lambda}^{n, k, \gamma}(0) \\
= & G_{\lambda}^{(n+\gamma)}(0)
\end{aligned}
$$

If $\sup _{\lambda>0} r_{\lambda}^{(n+\gamma)}(0)<\infty$, by Lemma 2.4, $\sup _{\lambda>0} r_{\lambda}^{(n)}(0)<\infty$ and $\sup _{\lambda>0} r_{\lambda}^{(k+\gamma)}(0)<\infty(0 \leq k<n)$ holds, so it follows from the result of case 1 that $E_{x}\left(T^{n+2}\right)<\infty$, hence the second term of the l.h.s of (3.2) is upper bounded in $\lambda>0$. For the third term

$$
\begin{aligned}
B_{\lambda}^{n, k, \gamma}(0) & =\frac{\gamma}{\Gamma(1-\gamma)} \int_{0}^{\infty} \frac{r_{\lambda+y}^{(k)}(0)\left(\varphi_{\lambda}^{(n-k)}(0)-\varphi_{\lambda+y}^{(n-k)}(0)\right)}{y^{\gamma+1}} d y \\
& \leq \sup _{\lambda>0} r_{\lambda}^{(k)}(0) \varphi_{\lambda}^{(n-k+\gamma)}(0) .
\end{aligned}
$$

Hence $\sup _{\lambda>0} G_{\lambda}^{(n+\gamma)}(0)<\infty$, which is equivalent to $E_{x}\left(T^{n+2+\gamma}\right)<\infty$ by Lemma 3.1.

Conversely supposing that $E_{x}\left(T^{n+2+\gamma}\right)<\infty$ we claim that $r_{\lambda}^{(n+\gamma)}(0)$ is bounded in $\lambda>0$. By the result of Case 1 , we know that $r_{\lambda}^{(n)}(0)$ is upper bounded in $\lambda>0$. So by Lemma 2.4 , it holds that $\sup _{\lambda>0} r_{\lambda}^{(k+\gamma)}(0)<$ 
$\infty(0 \leq k<n)$. Hence the second and third terms of the 1.h.s. in (3.2) are upper bounded in $\lambda>0$, which yields $\sup _{\lambda>0} r_{\lambda}^{(n+\gamma)}(0)<\infty$.

Finally using the inductive argument to (3.2) again, we can conclude that

$$
\lim _{\lambda \searrow 0} r_{\lambda}^{(n+\gamma)}(0)
$$

exists.

Case 3: $\alpha=2-\gamma(0<\gamma<1)$.

Using Lemma 2.2 (i) we have

$$
G_{\lambda}^{(-\gamma)}(0)=\frac{1}{\Gamma(\gamma)} \int_{0}^{\infty} y^{\gamma-1} r_{\lambda+y}(0) \varphi_{\lambda+y}(0) d y
$$

Note that $E_{x}\left(T^{2-\gamma}\right)<\infty$ is equivalent to the existence of $\lim _{\lambda \searrow 0} G_{\lambda}^{(-\gamma)}(0)$, which turns to

$$
\int_{0}^{\infty} y^{\gamma-1} r_{y}(0) \varphi_{y}(0) d y<\infty
$$

since $r_{\lambda}(0)>0(\lambda>0)$ holds by Lemma 2.4 , and (3.3) is equivalent to

$$
\lim _{\lambda \searrow 0} r_{\lambda}^{(-\gamma)}(0)=\frac{1}{\Gamma(\gamma)} \int_{0}^{\infty} y^{\gamma-1} r_{y}(0) d y<\infty .
$$

\section{$\S 4$. Proof of Theorem 1.2}

The main tool for the proof of Theorem 1.2 is Fourier analysis. For instance, applying Plancherel's identity to the function $r_{\lambda}^{(\alpha)}$ defined by (2.3), we have

$$
\left\|r_{\lambda}^{(\alpha)}\right\|_{2}^{2}=2 \pi \int_{0}^{\infty} e^{-2 \lambda t} t^{2 \alpha}\left(p_{t}(x, x)-\nu_{x}\right)^{2} d t<\infty,
$$

so that it holds

$$
\sup _{\lambda>0}\left\|r_{\lambda}^{(\alpha)}\right\|_{2}^{2}=2 \pi \int_{0}^{\infty} t^{2 \alpha}\left(p_{t}(x, x)-\nu_{x}\right)^{2} d t \leq \infty
$$

where $\|\cdot\|_{2}$ stands for the $L^{2}(\boldsymbol{R})$-norm, i.e.

$$
\|f\|_{2}^{2}=\int_{R}|f(z)|^{2} d z .
$$

Let us begin the following simple lemma. 
LEMmA 4.1. Let $X, Y$ be independent identically distributed positive random variables. If $E\left((X \wedge Y)^{\alpha}\right)<+\infty$ for $\alpha>0$, then $E\left(X^{\beta}\right)<+\infty$ holds for any $\beta \in\left(0, \frac{\alpha}{2}\right)$.

Proof. From the assumption it follows

$$
\int_{1}^{\infty} x^{\alpha-1} P(X \wedge Y \geq x) d x=\int_{1}^{\infty} x^{\alpha-1} P(X \geq x)^{2} d x<\infty .
$$

For $0<\beta<\alpha / 2$, use the Schwarz inequality to get

$$
\left(\int_{1}^{\infty} x^{\beta-1} P(X \geq x) d x\right)^{2} \leq \int_{1}^{\infty} x^{\alpha-1} P(X \geq x)^{2} d x \int_{1}^{\infty} x^{2 \beta-\alpha-1} d x<\infty
$$

which yields $E\left(X^{\beta}\right)<\infty$.

LEMMA 4.2. Let $\alpha>-1 / 2$. Suppose that

$$
\sup _{\lambda>0}\left\|r_{\lambda}^{(\alpha)}\right\|_{2}<\infty
$$

Then

$$
E_{x}\left(T^{\beta}\right)<\infty \text { for every } 0<\beta<\alpha+3 / 2 \text {. }
$$

Proof. Applying the Schwarz inequality we have

$$
\left(\int_{1}^{\infty} t^{\beta-2}\left|p_{t}(x, x)-\nu_{x}\right| d t\right)^{2} \leq \sup _{\lambda>0}\left\|r_{\lambda}^{(\alpha)}\right\|_{2}^{2} \int_{1}^{\infty} t^{2(\beta-\alpha-2)} d t<\infty,
$$

so that $\sup _{\lambda>0} r_{\lambda}^{(\beta-2)}(0)<\infty$ holds for $\beta>1$. Hence by virtue of Theorem 1.1 we get $(4.5)$.

Lemma 4.3. Let $G_{\lambda}^{(\beta)}(z)$ be the function defined by (2.5).

(i) $\operatorname{Let} \beta \geq 0$.

$$
\sup _{\lambda>0}\left\|G_{\lambda}^{(\beta)} / \varphi_{\lambda}\right\|_{2}<\infty
$$

holds if and only if

$$
E_{x} \otimes E_{x}\left(T T^{\prime}\left(T \wedge T^{\prime}\right)^{2 \beta+1}\right)<\infty .
$$

(ii) Let $-1 / 2<\beta<0$.

$$
\int_{0}^{\infty} \lambda^{-2 \beta-1}\left\|G_{\lambda} / \varphi_{\lambda}\right\|_{2}^{2} d \lambda<\infty
$$


holds if and only if

$$
E_{x} \otimes E_{x}\left(T T^{\prime}\left(T \wedge T^{\prime}\right)^{2 \beta+1}\right)<\infty .
$$

(iii) Let $\beta=-1 / 2$.

$$
\int_{0}^{1}\left\|G_{\lambda} / \varphi_{\lambda}\right\|_{2}^{2} d \lambda<\infty
$$

holds if and only if

$$
E_{x} \otimes E_{x}\left(T T^{\prime} \log _{+}\left(T \wedge T^{\prime}\right)\right)<\infty,
$$

where $\log _{+} x=\max \{\log x, 0\}$.

Proof. Note that if $\beta>-1 / 2, g(u) u^{\beta}$ is extended to an absolutely continuous function on $\boldsymbol{R}$ with value 0 on $(-\infty, 0]$ and $e^{-\lambda u} g(u) u^{\beta}$ and $\left(e^{-\lambda u} g(u) u^{\beta}\right)^{\prime}$ are $L^{2}$-function. Then Plancherel's identity and Lemma 2.3 (i) implies that for some $c_{1}>0$

$$
c_{1}\left\|G_{\lambda}^{(\beta)} / \varphi_{\lambda}\right\|_{2} \leq\left\|e^{-\lambda u} g(u) u^{\beta}\right\|_{2}+\left\|e^{-\lambda u}\left(g(u) u^{\beta}\right)^{\prime}\right\|_{2}
$$

for $0<\lambda \leq 1$, and that for some $c_{2}>0$

$$
\left\|e^{-\lambda u} g(u) u^{\beta}\right\|_{2} \leq c_{2}\left\|G_{\lambda}^{(\beta)} / \varphi_{\lambda}\right\|_{2} .
$$

As easily seen, if $\beta \geq 0$, then $\sup _{\lambda>0}\left\|e^{-\lambda u} g(u) u^{\beta}\right\|_{2}<\infty$ turns to

$$
E_{x} \otimes E_{x}\left(\int_{0}^{T \wedge T^{\prime}}(T-u)\left(T^{\prime}-u\right) u^{2 \beta} d u\right)<\infty
$$

which is equivalent to (4.6). Observing that $E_{x} \otimes E_{x}\left(\left(T \wedge T^{\prime}\right)^{2 \beta+1}\right)<\infty$ implies $\left\|e^{-\lambda u}\left(g(u) u^{\beta}\right)^{\prime}\right\|_{2}<\infty$, we obtain the conclusion of (i).

If $-1 / 2<\beta<0$, by (4.9) and (4.10), (4.7) turns to

$$
\int_{0}^{\infty} \lambda^{-2 \beta-1} \int_{0}^{\infty} e^{-2 \lambda u}\left(g(u)^{2}+g^{\prime}(u)^{2}\right) d u d \lambda<\infty,
$$

which is equivalent to

$$
\int_{0}^{\infty} u^{2 \beta} g(u)^{2} d u<\infty
$$

and further to (4.8). The case $\beta=-1 / 2$ is essentially the same as the previous case $-1 / 2<\beta<0$, so the proof is omitted. 
LEMMA 4.4. For $0 \leq k \leq n$ and $0<\gamma<1$, let $B_{\lambda}^{n, k, \gamma}(z)$ be the function defined by (2.10). Suppose that

$$
E_{x}\left(T^{n-k+1+\gamma}\right)<\infty \quad \text { and } \quad \sup _{\lambda>0}\left\|r_{\lambda}^{(k)}\right\|_{2}<\infty
$$

then it holds

$$
\sup _{\lambda>0}\left\|B_{\lambda}^{n, k, \gamma} / \varphi_{\lambda}\right\|_{2}<\infty
$$

Proof. Note that

(4.12) $\left\|B_{\lambda}^{n, k, \gamma} / \varphi_{\lambda}\right\|_{2} \leq \frac{\gamma}{\Gamma(1-\gamma)} \int_{0}^{\infty} \frac{\left\|r_{\lambda+y}^{(k)}\right\|_{2}}{y^{\gamma+1}}\left\|\frac{\varphi_{\lambda}^{(n-k)}-\varphi_{\lambda+y}^{(n-k)}}{\varphi_{\lambda}}\right\|_{\infty} d y$

Let

$$
f_{\lambda}(y)=\left\|\left(\varphi_{\lambda}^{(n-k)}-\varphi_{\lambda+y}^{(n-k)}\right) / \varphi_{\lambda}\right\|_{\infty} .
$$

Using Lemma 2.3 and integral by parts we see that for some constant $C>0$

$$
\begin{aligned}
f_{\lambda}(y) \leq & C \sup _{z \in R}\left|E_{x}\left(\int_{0}^{T}(1+\lambda+i z) e^{-(\lambda+i z) t}\left(1-e^{-y t}\right)\right) t^{n-k} d t\right| \\
\leq & C\left(E_{x}\left(\int_{0}^{T}\left(1-e^{-y t}\right) t^{n-k} d t\right)+E_{x}\left(\left(1-e^{-y T}\right) T^{n-k}\right)\right. \\
& \left.+E_{x}\left(\int_{0}^{T} y e^{-y t} t^{n-k} d t\right)+(n-k) E_{x}\left(\int_{0}^{T}\left(1-e^{-y t}\right) t^{n-k-1} d t\right)\right),
\end{aligned}
$$

so that for some $C_{1}>0$

$$
\int_{0}^{\infty} \frac{f_{\lambda}(y)}{y^{\gamma+1}} d y \leq C_{1}\left(E\left(T^{n-k+\gamma+1}\right)+E\left(T^{n-k+\gamma}\right)\right) .
$$

Hence (4.11) follows from (4.12), (4.13) and $\sup _{\lambda>0}\left\|r_{\lambda}^{(k)}\right\|_{2}<\infty$ by the assumption.

\section{Proof of Theorem 1.2.}

Case $1: \beta=2 n \quad\left(n \in \boldsymbol{Z}_{+}\right)$

Note that by Lemma 2.2

$$
r_{\lambda}^{(n)}=G_{\lambda}^{(n)} / \varphi_{\lambda}-\sum_{k=0}^{n-1}\left(\begin{array}{l}
n \\
k
\end{array}\right) r_{\lambda}^{(k)} \varphi_{\lambda}^{(n-k)} / \varphi_{\lambda}
$$


First we assume (1.5) with $\beta=2 n$. The it holds that

$$
\sup _{\lambda>0}\left\|r_{\lambda}^{(k)}\right\|_{2}<\infty \quad(0 \leq k \leq n)
$$

so by Lemma 4.2 we have

$$
E_{x}\left(T^{n+1}\right)<\infty
$$

from which and Lemma 2.3

$$
\sup _{\lambda>0}\left\|\varphi_{\lambda}^{(n-k)} / \varphi_{\lambda}\right\|_{\infty}<\infty \quad(0 \leq k \leq n-1) .
$$

Hence the second term of the r.h.s. of (4.14) is $L^{2}$-bounded in $\lambda>0$, so that

$$
\sup _{\lambda>0}\left\|G_{\lambda}^{(n)} / \varphi_{\lambda}\right\|_{2}<\infty
$$

yielding (1.4) by Lemma 4.3. Conversely, assuming (1.4) with $\beta=2 n$, by Lemma 4.3 we see that the first term of the r.h.s. of (4.14) is $L^{2}$-bounded in $\lambda>0$. Also, by Lemma 4.1 it holds

$$
E_{x}\left(T^{n+1}\right)<\infty
$$

Accordingly using Lemma 2.3 and induction we get

$$
\sup _{\lambda>0}\left\|r_{\lambda}^{(n)}\right\|_{2}<\infty
$$

which yields (1.5).

Case $2: \beta=2(n+\gamma) \quad\left(n \in \boldsymbol{Z}_{+}, 0<\gamma<1\right)$

By Lemma 2.2

$$
\begin{aligned}
r_{\lambda}^{(n+\gamma)}= & G_{\lambda}^{(n+\gamma)} / \varphi_{\lambda}-\sum_{k=0}^{n-1}\left(\begin{array}{l}
n \\
k
\end{array}\right) r_{\lambda}^{(k+\gamma)} \varphi_{\lambda}^{(n-k)} / \varphi_{\lambda} \\
& -\sum_{k=0}^{n}\left(\begin{array}{l}
n \\
k
\end{array}\right) B_{\lambda}^{n, k, \gamma} / \varphi_{\lambda} .
\end{aligned}
$$

Assume (1.5) with $\beta=2(n+\gamma)$. Then it holds that

$$
\sup _{\lambda>0}\left\|r_{\lambda}^{(k+\gamma)}\right\|_{2}<\infty \quad(0 \leq k \leq n),
$$


so by Lemma 4.2

$$
E_{x}\left(T^{n+1+\gamma}\right)<\infty
$$

from which and Lemma 2.3

$$
\sup _{\lambda>0}\left\|\varphi_{\lambda}^{(n-k+\gamma)} / \varphi_{\lambda}\right\|_{\infty}<\infty \quad(0 \leq k \leq n-1) .
$$

Hence the second term of the r.h.s. of (4.15) is $L^{2}$-bounded in $\lambda>0$. Furthermore, by Lemma 4.4 the last term of the r.h.s. of (4.15) is bounded in $\lambda>0$, so that

$$
\sup _{\lambda>0}\left\|G_{\lambda}^{(n+\gamma)} / \varphi_{\lambda}\right\|_{2}<\infty
$$

yielding (1.4). Conversely, assuming (1.4) with $\beta=2(n+\gamma)$, by Lemma 4.3 we see that the first term of the r.h.s. of (4.15) is bounded in $\lambda>0$. Also, by Lemma 4.1 it holds

$$
E_{x}\left(T^{n+1+\gamma}\right)<\infty
$$

Hence by Lemma 4.4 and Case 1 the last term of the r.h.s. of (4.15) is $L^{2}$ bounded in $\lambda>0$. Accordingly using Lemma 2.3 and induction we get

$$
\sup _{\lambda>0}\left\|r_{\lambda}^{(n+\gamma)}\right\|_{2}<\infty
$$

which yields (1.5).

Case $3: \beta=-\gamma(0<\gamma<1)$

Note that

$$
\int_{0}^{\infty} \lambda^{\gamma-1}\left\|r_{\lambda}\right\|_{2}^{2} d \lambda=\Gamma(\gamma) 2^{-\gamma}\left\|r_{0+}^{(-\gamma / 2)}\right\|_{2}^{2} .
$$

So by Lemma 2.2

$$
\sup _{\lambda>0}\left\|r_{\lambda}^{(-\gamma / 2)}\right\|_{2}<\infty
$$

is equivalent to

$$
\int_{0}^{\infty} \lambda^{\gamma-1}\left\|G_{\lambda} / \varphi_{\lambda}\right\|_{2}^{2} d \lambda<\infty
$$

Accordingly the desired conclusion follows from Lemma 4.3.

Case $4: \beta=-1$

Note that

$$
\int_{0}^{1}\left\|r_{\lambda}\right\|_{2}^{2} d \lambda=2 \pi \int_{0}^{\infty} \frac{1-e^{-2 t}}{2 t}\left(p_{t}(x, x)-\nu_{x}\right)^{2} d t
$$


and the finiteness of (4.16) is equivalent to (1.7). It is also equivalent to

$$
\int_{0}^{1}\left\|G_{\lambda} / \varphi_{\lambda}\right\|_{2}^{2} d \lambda<\infty
$$

which turns to (1.6) by Lemma 4.3. Thus the proof of Theorem 1.2 is completed.

\section{REFERENCES}

[AI] S. Aspandiiarov and R. Iasnogorodski, General criteria of integrability of functions of passage-times for non-negative stochastic processes and their applications, Teor. Veroyatnost. i Primenen, 43 (1998), 509-539.

[Ch] K.L.Chung, Markov Chains with Stationary Transition Probabilities, SpringerVerlag, Berlin, New York, 1960.

[CK] R. Carmona and A. Klein, Exponential moments for hitting times of uniformly ergodic Markov processes, Ann. Probab., 11 (1983), 648-655.

[L] J. Lamperti, Criteria for stochastic processes II: passage-time moments, J. Math. Anal. Appl., 7 (1963), 127-145.

[MW] M. Menshikov and R.J. Williams, Passage-time moments for continuous non-negative stochastic processes and applications, Adv. Appl. Prob., 28 (1996), 747-762.

[SS] A. Shimizu and T. Soshi, Positively recurrent Markov chains and the stepping stone model as a Fleming-Viot process, (submitted).

[TT] P. Tuominen and R.L. Tweedie, Subgeometric rates of convergence of f-ergodic Markov chains, Adv. Appl. Prob., 26 (1994), 775-798.

Tokuzo Shiga

Department of Mathematics

Tokyo Institute of Technology

Oh-okayama, Tokyo 152-8551

Japan

Akinobu Shimizu

Institute of Natural Sciences

Nagoya City University

Nagoya 467-8501

Japan

Takahiro Soshi

Department of Applied Mathematics

Yokohama National University

Yokohama 240-0067

Japan 